Magee, Jeffrey

[49]

\section{Expression profiling of androgen target genes in prostate cancer}

\author{
Jeffrey Magee, Sushama Patil \& Jeffrey Milbrandt
}

Washington University School of Medicine, St. Louis, Missouri, USA

Normal prostate epithelial cells are dependent on androgen for growth, differentiation and survival. A subset of prostate tumors is similarly dependent on androgen, and androgen ablation therapy typically results in the stasis or regression of these tumors. However, hormone-independent clones commonly arise following androgen ablation, and these clones typically display a more aggressive, less differentiated phenotype. Hormone-independent tumors retain androgen receptor (AR) expression. This observation suggests that these tumors may still require AR signaling, although in an androgen-independent manner. This hypothesis is supported by the observation that serum prostate-specific antigen levels increase in hormone-independent cancer despite the absence of androgen. We propose that additional AR targets are necessary for mediating tumor progression, in both hormonedependent and -independent states. We have stimulated androgen-responsive LAPC4 cells with androgens, and we have used microarrays to identify additional androgen targets. These targets include transcription factors, growth factors, signaling molecules and cytoskeletal and metabolic proteins. The in vivo androgen dependence of these targets has been confirmed in xenograft models. We are now using laser capture microdissection and the polymerase chain reaction with reverse transcription (Taqman) to quantify target gene expression in benign and malignant prostate epithelia from multiple patients. We are clustering tumor samples based on their AR target gene expression profiles in order to identify subsets of genes that are misexpressed in tumors relative to benign tissues, are expressed in androgen-independent tumors and correlate with biochemical failure of radical prostatectomy (as determined by rising prostate-specific antigen levels after surgery).

Mahadevappa, Mamatha

\section{Dissecting oral cancer through large-scale gene expression profiling of laser capture microdissection samples}

Mamatha Mahadevappa ${ }^{1}$, Ilias Alevizos², Hiroe Ohyama², Xue Zhang ${ }^{2}$, Yokho Kohno ${ }^{2}$, Marshall Posner ${ }^{3}$, George Gallagher' ${ }^{2}$, Bruce Donoff ${ }^{4}$, Randy Todd ${ }^{4}$, David Wong $^{2} \&$ Janet Warrington ${ }^{1}$

\section{${ }^{1}$ Affymetrix Inc., Santa Clara, California 95051, USA}

${ }^{2}$ Department of Oral Medicine and Diagnostic Sciences, Division of Oral Pathology, Harvard School of Dental Medicine, Boston, Massachusetts 02115, USA

${ }^{3}$ Department of Medicine, Division of Hematology-Oncology, Dana Farber Cancer Institute, Boston, Massachusetts 02115, USA

${ }^{4}$ Department of Oral and Maxillofacial Surgery, Massachusetts General Hospital, Boston, Massachusetts 02114, USA

We have combined laser capture microdissection (LCM) sample collection of oral epithelial tissue with microarray analysis to study gene expression in oral cancer. Matched normal and malignant oral epithelial samples were collected from five patients. Each tissue sample was reduced from millions of mixed normal and malignant cells to a homogenous sample of thousands of normal or malignant cells using LCM. A combination of the total RNA method and multiple rounds of in vitro transcription was used to derive sufficient quality and quantities of labeled targets. We hybridized labeled target on HuGeneFL arrays containing probes representing approximately 6,800 full-length human genes. We derived a list of can- didate genes using three analysis tools: GeneChip, GeneCluster and Matlab; 27 candidate genes were identified by all three methods. Several genes involved in cell proteolysis, migration and xenobiotic pathways were differentially expressed. Other genes with altered patterns included transcription factors, oncogenes and tumor suppressor genes, and differentiation markers. For several genes involved in known metastatic or invasion pathways, results were validated by quantitative polymerase chain recation with reverse transcription.

Mariani, Luigi

[51]

\section{P311 and DAP3: Glioblastoma invasion genes identified by laser capture microdissection, differential display and QRT-PCR}

Luigi Mariani ${ }^{1}$, Wendy McDonough ${ }^{1}$, Christian Beaudry ${ }^{1}$, Dominique Hoelzinger ${ }^{1}$, Elzbieta Kaczmarek ${ }^{1}$, Alf Giese ${ }^{2}$, Stephen Coons ${ }^{1}$, Rolf Seiler ${ }^{3}$, Kristen Ross ${ }^{1} \&$ Michael Berens ${ }^{1}$

${ }^{1}$ Barrow Neurological Institute, Phoenix, Arizona, USA

${ }^{2}$ University Hospital Eppendorf, Hamburg, Germany

${ }^{3}$ Inselspital Berne, Berne, Switzerland

To identify genes involved in local tumor cell invasion, we performed differential display using two RNA samples derived from glioblastoma multiforme (GBM) cells collected by laser capture microdissection. We harvested tumor cells $(20,000$ each) from the central core and the invasive rim of a human GBM specimen and identified neoplastic cells at the invasive edge of the tumor using morphological criteria (mainly nuclear atypia). We found differential expression of 50-60 complementary DNA bands between rim and core. Two sequences were markedly overexpressed in the tumor rim and showed strong homology to the $8-\mathrm{kD}$ polypeptide P311 (GI: 1244509) and the 46-kD Death Associated Protein-3 (DAP3; GI: 10368653). Overexpression (2- to 20-fold) of P311 and DAP3 in invasive cells was confirmed by quantitative polymerase chain recation with reverse transcription (QRT-PCR) in six of seven and four of four additional GBM specimens, respectively. We found 2-fold and 5- to 80-fold overexpression of P311 and DAP3, respectively, by QRT-PCR and western blot analysis in GBM cell lines stimulated to migrate on cell-derived extracellular matrix (ECM) proteins. Immunostaining of glioblastoma specimens confirmed the presence of P311 and DAP3 at the invasive edge of the tumor. Treatment with antisense oligodeoxynucleotides directed against P311 and DAP3 messenger RNA caused a dose-dependent reduction in the migration rate of GBM cells on different substrates. The P311 and DAP3 proteins are overexpressed in invading human glioblastoma cells in vivo and in glioblastoma cell lines grown on cell-derived extracellular matrix in vitro. These proteins play an active role in migration on laminin- and cell-derived extracellular matrix, where the cells have a reduced ability to undergo apoptosis. 